\title{
Compactness of the integration operator associated with a vector measure
}

\author{
by \\ S. Okada (Sydney), W. J. Ricker (Sydney and Eichstätt) \\ and L. Rodríguez-Piazza (Sevilla)
}

\begin{abstract}
A characterization is given of those Banach-space-valued vector measures $m$ with finite variation whose associated integration operator $I_{m}: f \mapsto \int f d m$ is compact as a linear map from $L^{1}(m)$ into the Banach space. Moreover, in every infinite-dimensional Banach space there exist nontrivial vector measures $m$ (with finite variation) such that $I_{m}$ is compact, and other $m$ (still with finite variation) such that $I_{m}$ is not compact. If $m$ has infinite variation, then $I_{m}$ is never compact.
\end{abstract}

1. Introduction and statement of results. Let $X$ be a (complex) Banach space with norm $\|\cdot\|$ and dual space $X^{\prime}$. Let $\Sigma$ be a $\sigma$-algebra of subsets of a nonempty set $\Omega$ and $m: \Sigma \rightarrow X$ be a vector measure, i.e., a $\sigma$-additive set function. Associated with $m$ is the Banach space $L^{1}(m)$ of all (equivalence classes of) $m$-integrable functions $f: \Omega \rightarrow \mathbb{C}$ together with the integration operator $I_{m}: L^{1}(m) \rightarrow X$ given by $f \mapsto \int_{\Omega} f d m$. The operator $I_{m}$ is always linear and continuous. Although vector measures $m$ and the Banach spaces $L^{1}(m)$ have received considerable attention since their conception (see $[1-4,6,7,11,14-17,23]$ and the references therein, for example), the same is not true of the integration operator $I_{m}$. This is somewhat surprising since, for example, such an important operator as the Fourier transform map $f \mapsto \widehat{f}$ from $L^{1}([-\pi, \pi])$ into $c_{0}(\mathbb{Z})$ is of the form $I_{m}$ for a suitable $c_{0}(\mathbb{Z})$-valued measure $m$ (see [18]). The same is also true for other kernel operators, such as those of Volterra type, for example [4, 20]. Or, the representation of cyclic Banach spaces is given via the integration operator with respect to a suitable vector measure [8] and so on. Whereas the weak compactness of integration operators $I_{m}$ has been systematically treated $[18,19]$, the same is not true of compactness, although some results

2000 Mathematics Subject Classification: Primary 28B05, 46G10, $47 \mathrm{~B} 05$.

Key words and phrases: vector measure, variation, integration operator, compact operator.

The research of the first author was supported by the Australian Research Council, and that of the third author was partially supported by DGICYT Grant \# PB 96-1327. 
for particular vector measures are known $[4,20]$. The aim of this paper is to present a systematic investigation of the compactness properties of integration operators. It is time to be more precise.

The variation $|m|$ of a vector measure $m: \Sigma \rightarrow X$ is the smallest $\sigma$-additive, nonnegative scalar measure on $\Sigma$ satisfying $\|m(E)\| \leq|m|(E)$ for $E \in \Sigma$. This is equivalent to the usual definition via the "partition process" [6, pp. 2-3]. The variation $|m|$ is called finite (resp. $\sigma$-finite) if it is a finite (resp. $\sigma$-finite) measure. It turns out always to be the case that $L^{1}(|m|) \subseteq L^{1}(m)$; see Section 2.

For the definition of Bochner integrals we refer to [6, Ch. II]. Let $\lambda$ : $\Sigma \rightarrow[0, \infty)$ be a finite measure and let $\mathcal{B}(\lambda, X)$ denote the space of all $X$-valued, Bochner $\lambda$-integrable functions on $\Omega$. Given $G \in \mathcal{B}(\lambda, X)$, the Bochner integral of $G$ over a set $E \in \Sigma$ (with respect to $\lambda$ ) is denoted by (B) $-\int_{E} G d \lambda$ and is an element of $X$. The indefinite Bochner $\lambda$-integral of $G$ is defined to be the vector measure $G \cdot \lambda$ on $\Sigma$ given by $E \mapsto(\mathrm{B})-\int_{E} G d \lambda$. We point out that the scalar function $\|G(\cdot)\|$ is always $\Sigma$-measurable and $\lambda$ integrable. Given a vector measure $m: \Sigma \rightarrow X$ with finite variation, if there exists $G \in \mathcal{B}(\lambda, X)$, necessarily unique, such that $m$ equals the indefinite Bochner $\lambda$-integral $G \cdot \lambda$, then $G$ is called the Radon-Nikodym derivative of $m$ with respect to $\lambda$ and we write $G=d m / d \lambda$. A function $H: \Omega \rightarrow X$ is said to have $\lambda$-essentially relatively compact range if there exists a $\lambda$-null set $E \in \Sigma$ such that $H(\Omega \backslash E)$ is relatively compact in $X$ (i.e. its closure is compact).

Our first theorem characterizes compactness of $I_{m}$. Its proof (and of the other results of this section) is given in Section 3.

Theorem 1. Let $X$ be a Banach space and $m: \Sigma \rightarrow X$ be a vector measure with finite variation. Then the integration operator $I_{m}: L^{1}(m) \rightarrow X$ is compact if and only if both of the following conditions hold:

(i) the measure $m$ has a Radon-Nikodym derivative $G=d m / d|m| \in$ $\mathcal{B}(|m|, X)$ with respect to $|m|$ (i.e., $m=G \cdot|m|)$,

(ii) the function $G$ has $|m|$-essentially relatively compact range in $X$.

In this case, the identity $L^{1}(m)=L^{1}(|m|)$ necessarily holds, and $I_{m} f=(\mathrm{B})-\int_{\Omega} f \cdot G d|m|$ for every $f \in L^{1}(m)$.

As an immediate consequence of the proof of Theorem 1 (see Section 3) we have the following useful fact.

Corollary 1.1. Let $X$ be a Banach space and $m: \Sigma \rightarrow X$ be a vector measure with finite variation. If $J: L^{1}(|m|) \rightarrow L^{1}(m)$ denotes the natural injection, then the integration operator $I_{m}: L^{1}(m) \rightarrow X$ is compact if and only if $I_{m} \circ J: L^{1}(|m|) \rightarrow X$ is compact. 
Condition (i) of Theorem 1 has received some attention in the literature and so it may be worthwhile to record some equivalent properties, namely:

(i) $^{\prime}$ Given any $E \in \Sigma$ with $|m|(E)>0$ there exists a $\Sigma$-measurable set $F \subseteq E$ with $|m|(F)>0$ such that

$$
\operatorname{Ave}_{F}(m):=\left\{\frac{m(H)}{|m|(H)}: H \in \Sigma, H \subseteq F \text { and }|m|(H)>0\right\}
$$

is relatively compact in $X$.

(i)" The integration operator $I_{m}$ restricted to the Banach space $L^{\infty}(|m|)$ $=L^{\infty}(m)$, equipped with the essential sup-norm, is an $X$-valued nuclear operator.

The equivalence (i) $\Leftrightarrow(\mathrm{i})^{\prime}$ is due to M. A. Rieffel; see [21, Theorem 4.1] for the formulation given above. The equivalence $(\mathrm{i}) \Leftrightarrow(\mathrm{i})^{\prime \prime}$ can be found in [21, Theorem 5.1], as a special case of [6, Theorem VI.4.4]. Further equivalences with condition (i) of Theorem 1 can be found in [21, Theorem 5.2], for example.

If $X$ is finite-dimensional and $m$ is any $X$-valued measure, then $I_{m}$ is necessarily compact. The existence of (nontrivial) compact integration operators in general Banach spaces $X$ is guaranteed by the following result.

Theorem 2. Let $X$ be an infinite-dimensional Banach space. Then there exists an $X$-valued vector measure $m$ such that $m$ has finite variation, the range of $m$ is not contained in any finite-dimensional subspace of $X$, and the integration operator $I_{m}$ is compact.

It is also the case, for vector measures of finite variation, that noncompact integration operators exist in every infinite-dimensional space.

Theorem 3. Let $X$ be an infinite-dimensional Banach space. Then there exists an $X$-valued vector measure $m$ with finite variation such that its integration operator $I_{m}: L^{1}(m) \rightarrow X$ is not compact. Furthermore, such an $m$ can be chosen to satisfy $L^{1}(|m|)=L^{1}(m)$ and have a Radon-Nikodým derivative $d m / d|m| \in \mathcal{B}(|m|, X)$.

Concerning arbitrary vector measures $m$, the determination of whether or not $I_{m}$ is compact reduces to the situation of finite variation.

Theorem 4. Let $X$ be a Banach space and $m$ be an $X$-valued vector measure. If the integration operator $I_{m}: L^{1}(m) \rightarrow X$ is compact, then $m$ must have finite variation.

If the integration operator $I_{m}$ is compact, then $m$ has finite variation (cf. Theorem 4), and hence, $L^{1}(m)=L^{1}(|m|)$ by Theorem 1 . Moreover, if $L^{1}(m)$ is infinite-dimensional, then $L^{1}(|m|)$ contains a complemented subspace isomorphic to $\ell^{1}$. This can be seen by decomposing the finite measure 
$|m|$ into the direct sum of its atomic part $|m|_{\text {a }}$ and its (disjointly supported) nonatomic part $|m|_{\text {na }}$ and then applying the argument (and discussion) from [5, pp. 201-202] to $|m|_{\mathrm{a}}$ if $|m|$ has infinitely many atoms, and to $|m|_{\text {na }}$ otherwise. So, Theorem 4 provides an alternative proof of the following (slightly more general) result due to G. Curbera [3, Claim, p. 3800].

Corollary 4.1. Let $X$ be a Banach space and $m$ be an $X$-valued vector measure such that its integration operator $I_{m}: L^{1}(m) \rightarrow X$ is compact and $L^{1}(m)$ is infinite-dimensional. Then $m$ has finite variation and $L^{1}(m)$ contains a complemented copy of $\ell^{1}$.

By Theorem 4, if a vector measure has infinite variation, then its integration operator is not compact. There are many examples of such measures. To see this, let $\lambda: \Sigma \rightarrow[0, \infty]$ be any infinite but $\sigma$-finite measure. For the definition of a function $G: \Omega \rightarrow X$ being Pettis $\lambda$-integrable we refer to [6, Ch. II, §3]. In this case, the indefinite Pettis integral of $G$ with respect to $\lambda$, namely, $E \mapsto(\mathrm{P})-\int_{E} G d \lambda$ for $E \in \Sigma$ (where the element $(\mathrm{P})-\int_{E} G d \lambda$ of $X$ denotes the Pettis integral of $G$ over $E$ with respect to $\lambda$ ) is an $X$ valued vector measure with $\sigma$-finite variation [10, Proposition 5.6(iv)]. So, let $G: \Omega \rightarrow X$ be any strongly measurable function (see [6, p. 41] for the definition) which is Pettis $\lambda$-integrable but not Bochner $\lambda$-integrable. Then its indefinite Pettis $\lambda$-integral has infinite but $\sigma$-finite variation. Whenever $X$ is infinite-dimensional and $\lambda$ is Lebesgue measure on the half line $[0, \infty)$ such a function $G:[0, \infty) \rightarrow X$ always exists; see the proof of $[21$, Theorem 3.3]. For a characterization of vector measures with $\sigma$-finite variation we refer to [22, Theorem 2.4]. In every infinite-dimensional Banach space there also exist vector measures with infinite but not $\sigma$-finite variation, and such measures can even be chosen to have relatively compact range, [23, p. 90]. See also $[9,12]$ for further information about such measures.

2. Preliminaries. Let $\Sigma$ be a $\sigma$-algebra of subsets of a nonempty set $\Omega$. Let $X$ be a Banach space and $m: \Sigma \rightarrow X$ be a vector measure. Given $x^{\prime} \in$ $X^{\prime}$, let $\left\langle m, x^{\prime}\right\rangle$ denote the complex measure $E \mapsto\left\langle m(E), x^{\prime}\right\rangle$; its variation $\left|\left\langle m, x^{\prime}\right\rangle\right|$ is then a finite measure. A $\Sigma$-measurable function $f: \Omega \rightarrow \mathbb{C}$ is called $m$-integrable if it is $\left\langle m, x^{\prime}\right\rangle$-integrable for all $x^{\prime} \in X^{\prime}$, and if there is a set function $\mathrm{fm}: \Sigma \rightarrow X$, necessarily unique, satisfying $\left\langle(\mathrm{fm})(E), x^{\prime}\right\rangle=$ $\int_{E} f d\left\langle m, x^{\prime}\right\rangle$ for all $x^{\prime} \in X^{\prime}$ and $E \in \Sigma$. Then the Orlicz-Pettis theorem ensures that $f m$ is also a vector measure. The classical notation $\int_{E} f d m:=$ $(\mathrm{fm})(E)$, for $E \in \Sigma$, will also be used. The vector space of all $m$-integrable functions is denoted by $L^{1}(m)$. Define a seminorm on $L^{1}(m)$ by

$$
\|f\|_{m}:=\sup \left\{\int_{\Omega}|f| d\left|\left\langle m, x^{\prime}\right\rangle\right|: x^{\prime} \in B\left[X^{\prime}\right]\right\}, \quad f \in L^{1}(m),
$$


where for any Banach space $Y$ we define $B[Y]:=\{y \in Y:\|y\| \leq 1\}$. The space $L^{1}(m)$ is then a complete seminormed space which contains the set of all $\mathbb{C}$-valued, $\Sigma$-simple functions as a dense subspace; see [14, Ch. IV] or [15, Theorem 2.4]. Associated with $m$ is its integration operator $I_{m}: L^{1}(m)$ $\rightarrow X$ defined by

$$
I_{m} f:=\int_{\Omega} f d m, \quad f \in L^{1}(m) .
$$

It is clear that $I_{m}$ is linear. Moreover, since $\left\|I_{m} f\right\| \leq\|f\|_{m}$ for $f \in L^{1}(m)$, the operator $I_{m}$ is also continuous.

For each set $E \in \Sigma$, its characteristic function is denoted by $\chi_{E}$. The semivariation $\|m\|$ of $m$ is the set function $\|m\|: \Sigma \rightarrow[0, \infty)$ defined by $\|m\|(E):=\left\|\chi_{E}\right\|_{m}$ for $E \in \Sigma$. In particular, $\|m\|$ is always finite (unlike the variation $|m|$, in general). It follows that $\|m\|(E) \leq|m|(E)$ for every $E \in \Sigma$ (see [6, Proposition I.1.11(a)]). An element $f \in L^{1}(m)$ is called $m$-null if $f m$ is the zero vector measure. This is equivalent to $\|f\|_{m}=0$. The quotient space of $L^{1}(m)$ modulo the $m$-null functions and equipped with the quotient norm induced by $\|\cdot\|_{m}$ is a Banach space; since no confusion will occur, we denote this quotient Banach space again by $L^{1}(m)$ and identify it with the seminormed space from which it arises (in the usual manner).

Sets $E \in \Sigma$ satisfying $\|m\|(E)=0$ are called $m$-null. The $m$-null and $|m|$-null sets coincide. This is immediate from the partition definition of $|m|$ (see $[6$, p. 2]) and the inequalities

$$
\|m(E)\| \leq\|m\|(E) \leq|m|(E), \quad E \in \Sigma .
$$

A property which holds outside an $m$-null set is said to hold $m$-almost everywhere (briefly, $m$-a.e.). A $\Sigma$-measurable function $f: \Omega \rightarrow \mathbb{C}$ is called $m$ essentially bounded if it is bounded $m$-a.e. The space of all such functions is denoted by $L^{\infty}(m)$ and is equipped with the essential sup-norm $\|\cdot\|_{\infty}$. It is known that $L^{\infty}(m) \subseteq L^{1}(m)$ and that, for each $f \in L^{\infty}(m)$,

$$
\left\|\int_{E} f d m\right\| \leq\|f\|_{\infty} \cdot\|m\|(E), \quad E \in \Sigma
$$

see [14, Theorem II.3.1] and [6, p. 6]. The quotient Banach space of $L^{\infty}(m)$ modulo the $m$-null functions is also denoted by $L^{\infty}(m)$. In particular, $L^{\infty}(|m|)=L^{\infty}(m)$.

For the following fact we refer to [6, Theorem II.2.4].

Lemma 2.1. Let $\lambda: \Sigma \rightarrow[0, \infty)$ be a finite measure and $X$ be a Banach space. If $G: \Omega \rightarrow X$ is a Bochner $\lambda$-integrable function, then its indefinite Bochner $\lambda$-integral $G \cdot \lambda: \Sigma \rightarrow X$ is a vector measure with finite variation given by

$$
|G \cdot \lambda|(E)=\int_{E}\|G(\omega)\| d \lambda(\omega), \quad E \in \Sigma .
$$


The next result, [6, Theorem III.2.2 \& p. 70], is one of the main tools of this paper.

Lemma 2.2. Let $\lambda: \Sigma \rightarrow[0, \infty)$ be a finite measure and $X$ be a $B a$ nach space. Then a continuous linear operator $T: L^{1}(\lambda) \rightarrow X$ is compact (i.e., the closure $\overline{T\left(B\left[L^{1}(\lambda)\right]\right)}$ is compact in $X$ ) if and only if there is a Bochner $\lambda$-integrable function $G \in \mathcal{B}(\lambda, X)$ with $\lambda$-essentially relatively compact range such that

$$
T f=(\mathrm{B})-\int_{\Omega} f(\omega) G(\omega) d \lambda(\omega), \quad f \in L^{1}(\lambda) .
$$

In this case, on the complement of some $\lambda$-null set the function $G$ takes its values in the closure (in $X)$ of $\left\{T f:\|f\|_{L^{1}(\lambda)}=1\right\}$.

Recall that the weak topology of a Banach space $X$ is determined by the saturated family of seminorms

$$
q_{F}(x):=\sum_{x^{\prime} \in F}\left|\left\langle x, x^{\prime}\right\rangle\right|, \quad x \in X,
$$

as $F$ varies through all finite subsets of $X^{\prime}$. The following notion will play a crucial role. A subset $W \subseteq X$ is said to be w-seminorm dominated (the "w" denotes "weak") if there exists a finite set $F \subseteq X^{\prime}$ such that

$$
\|x\| \leq q_{F}(x), \quad x \in W
$$

where $q_{F}$ is given by $(2.2)$.

ExAmple 2.3. (i) Every finite-dimensional subspace $Y$ of a Banach space $X$ is w-seminorm dominated. In fact, let $\operatorname{dim} Y=n$. Take a basis $\left\{e_{1}, \ldots, e_{n}\right\}$ of $Y$ and elements $\left\{x_{1}^{\prime}, \ldots, x_{n}^{\prime}\right\} \subseteq X^{\prime}$ such that $\left\langle e_{j}, x_{k}^{\prime}\right\rangle=\delta_{j k}$ for all $j, k \in\{1, \ldots, n\}$. Since the norm on $Y$ induced by $X$ is equivalent to the norm $y \mapsto \sum_{j=1}^{n}\left|\left\langle y, x_{j}^{\prime}\right\rangle\right|$ for $y \in Y$, it follows that $Y$ is w-seminorm dominated.

(ii) Let $\lambda: \Sigma \rightarrow[0, \infty]$ be any measure. Then the positive cone $W:=$ $\left\{f \in L^{1}(\lambda): f \geq 0\right\}$ of $X:=L^{1}(\lambda)$ is w-seminorm dominated. Indeed, the subset of $X^{\prime}$ consisting of the single function $\mathbb{1}$ (constantly equal to 1 on $\Omega$ ) satisfies

$$
\|f\|=\int_{\Omega}|f| d \lambda=\int_{\Omega} f d \lambda=\langle f, \mathbb{1}\rangle=|\langle f, \mathbb{1}\rangle|=q_{\{\mathbb{1}\}}(f), \quad f \in W .
$$

An important class of sets which are w-seminorm dominated is given by the following result.

Lemma 2.4. Let $X$ be a Banach space and $S[X]:=\{x \in X:\|x\|=1\}$ be its unit sphere. Then every relatively compact subset of $S[X]$ is w-seminorm dominated. 
Proof. Let $W \subseteq S[X]$ be a relatively compact set and define $U(x):=$ $\{y \in X:\|x-y\|<1 / 2\}$ for every $x \in \bar{W}$. The compact set $\bar{W} \subseteq S[X]$ includes finitely many points $x_{1}, \ldots, x_{n}$ satisfying $\bar{W} \subseteq \bigcup_{j=1}^{n} U\left(x_{j}\right)$. Fix $j \in\{1, \ldots, n\}$ and let $C_{j}$ denote the closed, convex hull of $\bar{W} \cap U\left(x_{j}\right)$ in $X$. Then $\|y\| \leq 3 / 2$ for all $y \in C_{j}$. Since $0 \notin C_{j}$, there is $x_{j}^{\prime} \in X^{\prime}$ such that

$$
\inf \left\{\left|\left\langle y, x_{j}^{\prime}\right\rangle\right|: y \in C_{j}\right\} \geq 3 / 2
$$

$x_{j}^{\prime}$ is the complexification of $u_{j} \in\left(X_{\mathbb{R}}\right)^{\prime}$, with $u_{j}$ suitably chosen as in [13, Theorem 7.3.4] for $A=\{0\}$ and $B=C_{j}$. Thus $\|y\| \leq 3 / 2 \leq\left|\left\langle y, x_{j}^{\prime}\right\rangle\right|$ for every $y \in C_{j}$. Consequently,

$$
\|x\| \leq \sum_{j=1}^{n}\left|\left\langle x, x_{j}^{\prime}\right\rangle\right|, \quad x \in W,
$$

because $W \subseteq \bar{W} \subseteq \bigcup_{j=1}^{n} C_{j}$.

The converse of the previous result fails in general.

Lemma 2.5. Let $X$ be an infinite-dimensional Banach space. Then there exists a subset of $S[X]$ which is w-seminorm dominated but not relatively compact.

Proof. Choose any basic sequence $\left\{x_{n}\right\}_{n=1}^{\infty}$ of unit vectors in $X$. That is, it is a Schauder basis for its closed linear span $Y$ in $X$ and there is a constant $K>0$ satisfying $\left\|\sum_{j=1}^{n} a_{j} x_{j}\right\| \leq K\left\|\sum_{j=1}^{k} a_{j} x_{j}\right\|$ for all choices of $n, k \in \mathbb{N}$ with $n<k$ and scalars $\left\{a_{j}\right\}_{j=1}^{k} \subseteq \mathbb{C}$ (see [5, Theorem V.1 \& Corollary V.3]). Given $n \geq 2$ choose any $a_{n+1}>0$ satisfying $\left\|\frac{1}{2} x_{1}+\frac{1}{4} x_{n}+a_{n+1} x_{n+1}\right\|=1$ and define $y_{n}:=\frac{1}{2} x_{1}+\frac{1}{4} x_{n}+a_{n+1} x_{n+1}$. Then, for all $n, k \in \mathbb{N}$ with $2 \leq n<k$, $\left\|y_{n}-y_{k}\right\|=\left\|\frac{1}{4} x_{n}+\left(a_{n+1} x_{n+1}-\frac{1}{4} x_{k}-a_{k+1} x_{k+1}\right)\right\| \geq K^{-1}\left\|\frac{1}{4} x_{n}\right\|=(4 K)^{-1}$. So, the subset $W:=\left\{y_{n}: n \geq 2\right\}$ of $S[X]$ is not relatively compact. To see that $W$ is w-seminorm dominated, choose any $\xi \in Y^{\prime}$ such that $\left\langle x_{1}, \xi\right\rangle=1$ and $\left\langle x_{j}, \xi\right\rangle=0$ for $j \geq 2$. By the Hahn-Banach theorem there is $x^{\prime} \in X^{\prime}$ which coincides with $\xi$ on $Y$. Since

$$
\left|\left\langle y_{n}, 2 x^{\prime}\right\rangle\right|=2\left|\left\langle y_{n}, x^{\prime}\right\rangle\right|=1=\left\|y_{n}\right\|, \quad n \geq 2,
$$

we see that $W$ is w-seminorm dominated.

Given a vector measure $m: \Sigma \rightarrow X$, we always have the inclusion $L^{1}(|m|) \subseteq L^{1}(m)$. Moreover, a function $f \in L^{1}(m)$ belongs to $L^{1}(|m|)$ if and only if its indefinite integral $f m: \Sigma \rightarrow X$ has finite variation, in which case $|f m|(E)=\int_{E}|f| d|m|$ (see [16, Theorem 4.2]). The natural inclusion $J: L^{1}(|m|) \rightarrow L^{1}(m)$ is continuous because

$$
\|f\|_{m} \leq|f m|(\Omega)=\int_{\Omega}|f| d|m|=\|f\|_{L^{1}(|m|)}, \quad f \in L^{1}(|m|) .
$$


In the case when $L^{1}(m)=L^{1}(|m|)$ as vector spaces, the open mapping theorem implies that the Banach spaces $L^{1}(m)$ and $L^{1}(|m|)$ are isomorphic. If $|m|(\Omega)=\infty$, then the inclusion $L^{1}(|m|) \subseteq L^{1}(m)$ is always proper as $\mathbb{1} \in L^{1}(m) \backslash L^{1}(|m|)$. Even if $|m|(\Omega)<\infty$, this inclusion may be proper. Indeed, such measures $m$ exist in every infinite-dimensional Banach space [18, Remark 2(d)].

Given a function $G: \Omega \rightarrow X$ and $x^{\prime} \in X^{\prime}$, let $\left\langle G(\cdot), x^{\prime}\right\rangle$ denote the scalar-valued function $\omega \mapsto\left\langle G(\omega), x^{\prime}\right\rangle$ for $\omega \in \Omega$.

Lemma 2.6. Let $X$ be a Banach space and $m: \Sigma \rightarrow X$ be a vector measure with finite variation.

(i) If there exists a nonempty finite set $F \subseteq X^{\prime}$ such that

$$
|m|(E) \leq \sum_{x^{\prime} \in F}\left|\left\langle m, x^{\prime}\right\rangle\right|(E), \quad E \in \Sigma,
$$

then necessarily $L^{1}(m)=L^{1}(|m|)$.

(ii) Suppose that there exists a finite measure $\lambda: \Sigma \rightarrow[0, \infty)$ with the property that $m$ has a Radon-Nikodym derivative $G \in \mathcal{B}(\lambda, X)$ with respect to $\lambda($ i.e., $m=G \cdot \lambda)$. Then there exists an m-null set $E \in \Sigma$ such that $G(\Omega \backslash E) \subseteq X$ is w-seminorm dominated if and only if (2.3) holds for some nonempty finite set $F \subseteq X^{\prime}$.

Proof. (i) This is an immediate consequence of the inclusion $L^{1}(|m|) \subseteq$ $L^{1}(m)$ and $(2.3)$ since

$$
L^{1}(|m|) \subseteq L^{1}(m) \subseteq \bigcap_{x^{\prime} \in F} L^{1}\left(\left|\left\langle m, x^{\prime}\right\rangle\right|\right) \subseteq L^{1}(|m|) .
$$

(ii) It follows from Lemma 2.1 that $\left|\left\langle m, x^{\prime}\right\rangle\right|=\left|\left\langle G(\cdot), x^{\prime}\right\rangle\right| \cdot \lambda$ on $\Sigma$ for all $x^{\prime} \in F$, and $|m|=\|G(\cdot)\| \cdot \lambda$ on $\Sigma$. Therefore (2.3) holds if and only if

$$
\int_{E}\|G(\omega)\| d \lambda(\omega) \leq \int_{E} \sum_{x^{\prime} \in F}\left|\left\langle G(\omega), x^{\prime}\right\rangle\right| d \lambda(\omega), \quad E \in \Sigma,
$$

which is the case if and only if $\|G(\omega)\| \leq \sum_{x^{\prime} \in F}\left|\left\langle G(\omega), x^{\prime}\right\rangle\right|$ for $m$-almost every $\omega \in \Omega$.

REMARK 2.7. (i) If the range of a vector measure $m: \Sigma \rightarrow X$ is w-seminorm dominated, then $m$ has finite variation and satisfies (2.3) for some nonempty finite set $F \subseteq X^{\prime}$. In particular, $L^{1}(m)=L^{1}(|m|)$.

The converse is false in general, i.e. there exists a vector measure $m$ (it can even be chosen with $I_{m}$ compact!) such that (2.3) holds for some nonempty finite set $F \subseteq X^{\prime}$ but the range of $m$ is not w-seminorm dominated. Indeed, let $X:=\ell^{2}$ and let $\Sigma$ denote the $\sigma$-algebra of all Borel subsets of $\Omega:=[0,2]$. Let $\alpha_{n}>0$ for $n=2,3, \ldots$ be any sequence decreasing to 0 and, for each $n \geq 1$, let $A_{n}:=[1 /(n+1), 1 / n)$. Define $G: \Omega \rightarrow X$ by 


$$
G(\omega):=-\chi_{[1,2]}(\omega) \cdot e_{1}+\sum_{n=1}^{\infty} \chi_{A_{n}}(\omega) \cdot\left(e_{1}+\alpha_{n+1} e_{n+1}\right), \quad \omega \in \Omega,
$$

where $\left\{e_{n}\right\}_{n=1}^{\infty}$ is the standard orthonormal basis of $X$. Since $\left\langle G(\cdot), e_{1}\right\rangle=$ $\chi_{(0,1)}(\cdot)-\chi_{[1,2]}(\cdot)$ and $\|G(\omega)\| \leq\left(1+\alpha_{2}^{2}\right)^{1 / 2}$ for every $\omega \in \Omega$, it follows that

$$
\|G(\omega)\| \leq\left|\left\langle G(\omega),\left(1+\alpha_{2}^{2}\right)^{1 / 2} e_{1}\right\rangle\right|, \quad \omega \in \Omega,
$$

i.e. the range of $G$ is w-seminorm dominated. But $G$ is easily seen to be Bochner integrable with respect to Lebesgue measure $\lambda$ on $\Sigma$. Hence, if we let $m:=G \cdot \lambda$, then it follows from Lemma 2.6(ii) that $m$ satisfies (2.3) with $F:=\left\{\left(1+\alpha_{2}^{2}\right)^{1 / 2} e_{1}\right\}$.

For each $n \geq 1$, let $B_{n}:=((n+2) /(n+1),(n+1) / n)$ and note that $m\left(A_{n} \cup B_{n}\right)=\lambda\left(A_{n}\right) \alpha_{n+1} e_{n+1}$. Suppose that there exists a nonempty finite set $H \subseteq X^{\prime}$ such that

$$
\|m(E)\| \leq \sum_{x^{\prime} \in H}\left|\left\langle m(E), x^{\prime}\right\rangle\right|, \quad E \in \Sigma .
$$

By choosing $E$ to be $A_{n} \cup B_{n}$ it would follow that $1 \leq \sum_{x^{\prime} \in H}\left|\left\langle e_{n+1}, x^{\prime}\right\rangle\right|$ for every $n \in \mathbb{N}$, which is impossible. So, $m(\Sigma)$ is not w-seminorm dominated.

Since $d m / d|m|$ is the function $\omega \mapsto G(\omega) /\|G(\omega)\|$ for $\omega \in \Omega$, and this function has $|m|$-essential range equal to the relatively compact subset $\left\{-e_{1}\right\} \cup\left\{\left(1+\alpha_{n+1}^{2}\right)^{-1 / 2}\left(e_{1}+\alpha_{n+1} e_{n+1}\right): n \in \mathbb{N}\right\}$ of $S[X]$, it follows from Theorem 1 that $I_{m}$ is compact.

(ii) The converse of Lemma 2.6(i) is not valid in general. To see this, let $X:=c_{0}$ and consider the functions

$$
g_{n}:=r_{n} \chi_{A_{n}}, \quad n \in \mathbb{N},
$$

where $A_{n}:=[0,1-1 /(n+1)]$ and $\left\{r_{n}\right\}_{n=1}^{\infty} \subseteq L^{\infty}([0,1])$ is the sequence of Rademacher functions. Since $\left\{g_{n}\right\}_{n=1}^{\infty}$ is a weak-star null sequence in the dual space $L^{\infty}([0,1])$ of $L^{1}([0,1])$, it is clear that the set function $m: \Sigma \rightarrow X$ defined by

$$
m(E):=\left(\int_{E} g_{n} d \lambda\right)_{n=1}^{\infty} \in c_{0}, \quad E \in \Sigma,
$$

is a vector measure, where $\Sigma$ is the $\sigma$-algebra of all Borel subsets of $\Omega:=$ $[0,1]$ and $\lambda$ is Lebesgue measure on $\Sigma$. From the fact that $\left|r_{n}(\omega)\right|=1$ for all $\omega \in \Omega$ and $n \in \mathbb{N}$, it follows that $m$ has finite variation given by $|m|=\lambda$. Let $\left\{e_{n}^{\prime}\right\}_{n=1}^{\infty} \subseteq X^{\prime}=\ell^{1}$ be the standard basis of $\ell^{1}$. For each $f \in L^{1}(m)$ we have

$$
\int_{0}^{1-1 /(n+1)}|f| d \lambda=\int_{0}^{1}|f| d\left|\left\langle m, e_{n}^{\prime}\right\rangle\right| \leq\|f\|_{m}, \quad n \in \mathbb{N} .
$$

The monotone convergence theorem implies that $\int_{0}^{1-1 /(n+1)}|f| d \lambda \rightarrow\|f\|_{L^{1}(\lambda)}$ as $n \rightarrow \infty$, from which it is clear that $L^{1}(m)=L^{1}(|m|)$ with equality 
of norms. If $x^{\prime}=\left(x_{n}^{\prime}\right)_{n=1}^{\infty} \in X^{\prime}$, then $d\left|\left\langle m, x^{\prime}\right\rangle\right| / d \lambda \leq \sum_{n=1}^{\infty}\left|x_{n}^{\prime}\right| \cdot\left|g_{n}(\cdot)\right|$ pointwise a.e. on $\Omega$. So, if (2.3) holds for some nonempty finite set $F \subseteq X^{\prime}$, then there exists $\xi=\left(\xi_{n}\right)_{n=1}^{\infty} \in X^{\prime}$, with $\xi_{n}>0$ for all $n \in \mathbb{N}$, such that

$$
\sum_{x^{\prime} \in F}\left|\left\langle m, x^{\prime}\right\rangle\right|(E) \leq \sum_{n=1}^{\infty} \xi_{n} \int_{E}\left|g_{n}(\omega)\right| d \lambda(\omega), \quad E \in \Sigma .
$$

Choosing $E:=[1-1 / k, 1]$ gives

$$
\sum_{x^{\prime} \in F}\left|\left\langle m, x^{\prime}\right\rangle\right|([1-1 / k, 1]) \leq \sum_{n \geq k} \xi_{n} \cdot \lambda([1-1 / k, 1]), \quad k \in \mathbb{N} .
$$

It then follows from the identity $|m|=\lambda$ and (2.3) that

$$
\lambda([1-1 / k, 1])=|m|([1-1 / k, 1]) \leq \sum_{n \geq k} \xi_{n} \cdot \lambda([1-1 / k, 1]), \quad k \in \mathbb{N},
$$

which is impossible as $\xi \in \ell^{1}$. So, there is no nonempty finite set $F \subseteq X^{\prime}$ such that (2.3) holds.

We conclude with the following result needed later.

LEMma 2.8. Let $Y$ be a Banach space and $Z$ be a closed subspace of $Y$ having finite codimension. For each $\varepsilon>0$ there exists a finite set $F_{\varepsilon} \subseteq B[Y]$ such that

$$
B[Y] \subseteq(1+\varepsilon)\left(\mathrm{bco}\left(F_{\varepsilon}\right)+2 B[Z]\right),
$$

where $\mathrm{bco}(H)$ denotes the balanced convex hull of any set $H \subseteq Y$.

Proof. Let $\delta:=\varepsilon /(2(1+\varepsilon))$. For $y \in Y$, let $\widetilde{y}$ denote its equivalence class in the quotient Banach space $Y / Z$ which is equipped with the quotient norm $\|\cdot\|_{Y / Z}$. Since $0<\delta<1 / 2$ and $\operatorname{dim}(Y / Z)<\infty$, there are finitely many (say $N$ ) open balls of radius $\delta$, having their centres in the open unit ball of $Y / Z$, which cover $B[Y / Z]$. So, there exists a finite set $F_{\varepsilon}:=\left\{w_{1}, \ldots, w_{N}\right\} \subseteq$ $B[Y]$ with the property that for every $y \in B[Y]$ there is $j(y) \in\{1, \ldots, N\}$ such that $\left\|\widetilde{y}-\left(w_{j(y)}\right)^{\sim}\right\|_{Y / Z}<\delta$, and hence, there is also $z \in Z$ (depending on $y$ ) such that

$$
\left\|y-z-w_{j(y)}\right\|<\delta .
$$

Observe that $z$ also satisfies $\|z\| \leq 1+\delta+\|y\|$. Define a closed, balanced, convex set $G \subseteq Y$ by $G:=2 B[Z]+\operatorname{bco}\left(F_{\varepsilon}\right)$. Then we have just established that for every $y \in(1-\delta) B[Y] \subseteq B[Y]$ there exists $x \in G$ (depending on $y$ ) with $\|y-x\|<\delta$. Indeed, the choice $x:=z+w_{j(y)}$ as in (2.4) has the desired property since $\|z\| \leq 1+\delta+\|y\| \leq 1+\delta+(1-\delta)=2$ shows that $z \in 2 B[Z]$.

Fix $y^{\prime} \in Y^{\prime}$. Then we have

$$
\begin{aligned}
\sup \left\{\left|\left\langle y, y^{\prime}\right\rangle\right|: y \in(1-2 \delta) B[Y]\right\} & \\
& =\sup \left\{\left|\left\langle y, y^{\prime}\right\rangle\right|: y \in(1-\delta) B[Y]\right\}-\delta\left\|y^{\prime}\right\| .
\end{aligned}
$$


But, for each $y \in(1-\delta) B[Y]$, choose a vector $x_{y} \in G$ satisfying $\left\|y-x_{y}\right\|<\delta$. Then

$$
\left|\left\langle y, y^{\prime}\right\rangle\right| \leq\left|\left\langle y-x_{y}, y^{\prime}\right\rangle\right|+\left|\left\langle x_{y}, y^{\prime}\right\rangle\right| \leq \delta\left\|y^{\prime}\right\|+\sup \left\{\left|\left\langle x, y^{\prime}\right\rangle\right|: x \in G\right\},
$$

from which it is clear that

$$
\sup \left\{\left|\left\langle y, y^{\prime}\right\rangle\right|: y \in(1-\delta) B[Y]\right\} \leq \delta\left\|y^{\prime}\right\|+\sup \left\{\left|\left\langle x, y^{\prime}\right\rangle\right|: x \in G\right\} .
$$

Then (2.5) implies

$$
\begin{aligned}
\sup \left\{\left|\left\langle y, y^{\prime}\right\rangle\right|: y \in\right. & B[Y]\} \\
& \leq \sup \left\{\left|\left\langle x, y^{\prime}\right\rangle\right|: x \in(1-2 \delta)^{-1} G\right\}, \quad y^{\prime} \in Y^{\prime} .
\end{aligned}
$$

It follows that

$$
B[Y] \subseteq(1-2 \delta)^{-1} G
$$

In fact, assume the contrary. Then there would exist a vector $y_{0} \in B[Y] \backslash$ $(1-2 \delta)^{-1} G$. Since $\left\{y_{0}\right\}$ is compact and convex and since $(1-2 \delta)^{-1} G$ is balanced, convex and closed, by [13, Corollary 5, p. 131] there would exist $y_{0}^{\prime} \in Y^{\prime}$ satisfying

$$
\sup \left\{\left|\left\langle x, y_{0}^{\prime}\right\rangle\right|: x \in(1-2 \delta)^{-1} G\right\}<\left|\left\langle y_{0}, y_{0}^{\prime}\right\rangle\right| .
$$

But this contradicts the inequality

$$
\left|\left\langle y_{0}, y_{0}^{\prime}\right\rangle\right| \leq \sup \left\{\left|\left\langle x, y_{0}^{\prime}\right\rangle\right|: x \in(1-2 \delta)^{-1} G\right\}
$$

which follows from (2.6) because $y_{0} \in B[Y]$. Thus (2.7) holds, and hence,

$$
B[Y] \subseteq(1-2 \delta)^{-1} G=(1+\varepsilon) G=(1+\varepsilon)\left(\mathrm{bco}\left(F_{\varepsilon}\right)+2 B[Z]\right) .
$$

The previous result is optimal in the sense that there exist a Banach space $Y$ and a closed subspace $Z$ of $Y$ (with finite codimension) having the property that if

$$
B[Y] \subseteq K+\beta B[Z]
$$

for any compact set $K \subseteq Y$, then necessarily $\beta>2$. Moreover, if (2.8) holds for some $K$ of the form $K=\alpha \operatorname{bco}(F)$ with $F$ a finite subset of $B[Y]$, then necessarily $\alpha>1$. Indeed, take $Y:=\ell^{1}$ and $Z:=\operatorname{ker}(\psi)$, where $\psi \in\left(\ell^{1}\right)^{\prime}$ is the linear functional given by

$$
\langle y, \psi\rangle:=\sum_{n=1}^{\infty}\left(1-n^{-1}\right) y_{n}, \quad y=\left(y_{n}\right)_{n=1}^{\infty} \in Y .
$$

The point is that $\|\psi\|=1$ but $|\langle y, \psi\rangle|<1$ for every $y \in B[Y]$.

Suppose that there exist $\beta>0$ and a compact set $K \subseteq Y$ such that (2.8) holds. It can be seen (argue by contradiction) that there exists $\varepsilon>0$ with the property that $|\langle y, \psi\rangle| \leq 1-\varepsilon$ for all $y \in K \cap(1+\varepsilon) B[Y]$. Choose any $n_{0} \in \mathbb{N}$ satisfying

$$
\left|y_{j}\right|<\varepsilon / 3 \quad \text { for all } y \in K \text { and } j \geq n_{0} .
$$


Now choose an integer $n>n_{0}$ such that $n^{-1}<\varepsilon$ and let $e_{n} \in B[Y]$ be the corresponding $n$th standard basis vector of $Y$. By (2.8) there are $y \in K$ and $z \in B[Z]$ such that $e_{n}=y+\beta z$. Then

$$
1-\varepsilon<1-n^{-1}=\left\langle e_{n}, \psi\right\rangle=\langle y, \psi\rangle
$$

and, by the above property of $\varepsilon$, we have $\|y\|>1+\varepsilon$. It follows that $\beta>2$ since

$$
\begin{aligned}
\beta \geq\|\beta z\| & =\left\|e_{n}-y\right\|=\left|1-y_{n}\right|+\sum_{j \in \mathbb{N} \backslash\{n\}}\left|y_{j}\right| \\
& =\|y\|-\left|y_{n}\right|+\left|1-y_{n}\right|>1+\varepsilon+1-2\left|y_{n}\right| \\
& >2+\varepsilon-(2 \varepsilon / 3)>2 .
\end{aligned}
$$

Suppose now that (2.8) holds for some $\beta>2$ and $K$ of the form $K:=$ $\alpha \operatorname{bco}(F)$ with $0<\alpha \leq 1$ and $F$ a finite subset of $B[Y]$. Then $K \subseteq B[Y]$ and, by compactness, there exists $u \in K$ such that

$$
\sup \{|\langle y, \psi\rangle|: y \in K\}=|\langle u, \psi\rangle|<1 .
$$

But this is a contradiction since

$$
\begin{aligned}
1 & =\|\psi\| \leq \sup \{|\langle y, \psi\rangle|: y \in K\}+\sup \{|\langle y, \psi\rangle|: y \in \beta B[Z]\} \\
& =\sup \{|\langle y, \psi\rangle|: y \in K\}<1 .
\end{aligned}
$$

Accordingly, we must have $\alpha>1$.

3. Proofs of Theorems 1-4. The aim of this final section is to give the proofs of the theorems listed in Section 1 and to discuss some relevant examples.

Proof of Theorem 1. If $I_{m}$ is compact, then so is $I_{m} \circ J: L^{1}(|m|) \rightarrow X$, where $J: L^{1}(|m|) \rightarrow L^{1}(m)$ is the natural injection. By Lemma 2.2 there is $G \in \mathcal{B}(|m|, X)$ satisfying condition (ii) of Theorem 1 such that

$$
\left(I_{m} \circ J\right) f=(\mathrm{B})-\int_{\Omega} f \cdot G d|m|, \quad f \in L^{1}(|m|) .
$$

Since $\left(I_{m} \circ J\right) f=\int_{\Omega} f d m$ for every $f \in L^{1}(|m|)$, we see upon substituting $f=\chi_{E}$ for $E \in \Sigma$ that $m=G \cdot|m|$, i.e., condition (i) of Theorem 1 also holds.

Conversely, assume conditions (i) and (ii) of Theorem 1 are satisfied. Then (i) yields $|m|=\|G(\cdot)\| \cdot|m|$ (via Lemma 2.1). In particular, $I_{m} \circ J$ is compact from $L^{1}(|m|)$ into $X$; see Lemma 2.2. If $\mu: \Sigma \rightarrow[0, \infty)$ is any finite measure and $f \geq 0$ is any $\mu$-integrable function satisfying $\mu(E)=\int_{E} f d \mu$ for all $E \in \Sigma$, then it is routine to check that $f=\mathbb{1}$ ( $\mu$-a.e.). With $\mu:=|m|$ and $f:=\|G(\cdot)\|$ we conclude that $G(\omega) \in S[X]$ for $|m|$-almost every $\omega \in \Omega$. So, we assume that the range of $G$ lies within $S[X]$ and hence, by condition (ii) and Lemma 2.4, the range of $G$ is w-seminorm dominated. Now Lemma 2.6 
ensures that $L^{1}(|m|)=L^{1}(m)$. Since $J$ is then the identity operator and $I_{m} \circ J=I_{m}$, the compactness of $I_{m}$ follows from that of $I_{m} \circ J$.

Some additional comments concerning Theorem 1 are in order. If $X$ has the Radon-Nikodým property and $m: \Sigma \rightarrow X$ has finite variation, then condition (i) of Theorem 1 is automatically satisfied. Accordingly, the compactness of $I_{m}$ is then solely determined by whether or not $d m / d|m| \in$ $\mathcal{B}(|m|, X)$ has $|m|$-essentially relatively compact range in $X$. This is not always the case.

EXAMPLE 3.1. Let $\Omega:=[0,1]$ and $\Sigma$ be the $\sigma$-algebra of all Borel subsets of $\Omega$. Fix $p \in(1, \infty)$, in which case the reflexive Banach space $X:=L^{p}([0,1])$ has the Radon-Nikodým property [6, p. 218]. Define $m: \Sigma \rightarrow X$ by

$$
m(E): t \mapsto \int_{0}^{t} \chi_{E}(s) d s, \quad t \in[0,1],
$$

for $E \in \Sigma$. Then $m$ is a vector measure with finite variation and $|m|(E)=$ $\int_{E}(1-s)^{1 / p} d s$ for every $E \in \Sigma$. Moreover, $G=d m / d|m|$ is the function $G(s)=(1-s)^{-1 / p} \cdot \chi_{[s, 1]}(\cdot)$ for $s \in \Omega$. It is shown in the proof of [20, Proposition 5.2(ii)] that $G$ (called $h_{p}$ in [20]) does not have $|m|$-essentially relatively compact range, and hence $I_{m}$ is not compact.

A similar phenomenon to that in Example 3.1 can occur in spaces without the Radon-Nikodým property.

EXAmple 3.2. Let $\Omega$ and $\Sigma$ be as in Example 3.1. Then $X:=L^{1}([0,1])$ does not have the Radon-Nikodým property [6, p. 219]. Define a vector measure $m: \Sigma \rightarrow X$ again by the formula (3.1), in which case $m$ has finite variation given by $|m|(E)=\int_{E}(1-s) d s$ for $E \in \Sigma$. It is shown in [18, Lemma 2.1] that $G:=d m / d|m|$ exists and is the $X$-valued function $G(s)=(1-s)^{-1} \chi_{[s, 1]}(\cdot)$ for $s \in \Omega$. Since $I_{m}$ is not even weakly compact [18, Proposition 2.7], it cannot be compact. By Theorem 1, $G$ does not have $|m|$-essentially relatively compact range.

For examples of vector measures of finite variation which have no RadonNikodým derivative with respect to their variation (i.e. condition (i) of Theorem 1 fails) we refer to the Volterra measures considered in [20] in the spaces $C([0,1])$ and $L^{\infty}([0,1])$. Neither of these spaces has the Radon-Nikodým property [6, p. 219].

REMARK 3.3. (i) The vector measure $m$ of Example 3.2 satisfies $L^{1}(m)$ $=L^{1}(|m|)$; see [18, Lemma 2.4], or use Example 2.3(ii) and Remark 2.7. This shows that the compactness of $I_{m}$ is not equivalent to the equality $L^{1}(m)=L^{1}(|m|)$; see the statement of Theorem 1 . 
(ii) The compactness of $I_{m}$ clearly implies the relative compactness of $m(\Sigma)$ in $X$. The converse is not true in general. To see this let $X:=$ $L^{p}([0, \infty])$ for any $1 \leq p \leq \infty$ and define $m$ as in Examples 3.1 and 3.2. It is known that the classical Volterra operator $V: X \rightarrow X$ defined by $V f: t \mapsto \int_{0}^{t} f(s) d s$, for $t \in[0,1]$ and every $f \in X$, is a compact operator. Since $m(E)=V\left(\chi_{E}\right)$ for all $E \in \Sigma$, it follows that $m(\Sigma)$ is relatively compact in $X$.

Proof of Theorem 2. Let $\Omega:=[0,1]$ and $\lambda$ be Lebesgue measure on the Borel $\sigma$-algebra $\Sigma$ of $\Omega$. Choose any basic sequence $\left\{x_{n}\right\}_{n=1}^{\infty}$ of unit vectors in $X$ (see [5, Corollary V.3]). By using the fact that the sequence $\left\{n^{-3} e^{i n \omega} x_{n}\right\}_{n=1}^{\infty}$ is absolutely summable in $X$ for every $\omega \in \Omega$, we can define a function $H: \Omega \rightarrow X$ by $H(\omega):=\sum_{n=1}^{\infty} n^{-3} e^{i n \omega} x_{n}$ for $\omega \in \Omega$. Then $H$ is continuous because $\|H(\omega)-H(u)\| \leq|\omega-u|\left(\sum_{n=1}^{\infty} n^{-2}\right)$ whenever $u, \omega \in \Omega$. Moreover, $H(\omega) \neq 0$ for each $\omega \in \Omega$. This is a consequence of the fact that $\left\{x_{n}\right\}_{n=1}^{\infty}$ is a Schauder basis for its closed linear span $Y$ and the fact that $H(\omega)=0$ if and only if $n^{-3} e^{i n \omega}=0$ for every $n \in \mathbb{N}$, which never occurs. Clearly $\left\langle H(\cdot), x^{\prime}\right\rangle$ is $\Sigma$-measurable for each $x^{\prime} \in X^{\prime}$. Since $H$ takes its values in the separable subspace $Y$ of $X$, it follows from the Pettis measurability theorem $[6$, p. 42$]$ that $H$ is strongly measurable. Accordingly, $H \in \mathcal{B}(\lambda, X)$. Let $m:=H \cdot \lambda$ be the indefinite Bochner $\lambda$-integral of $H$ with respect to $\lambda$. By Lemma 2.1, $m$ has finite variation $|m|=\|H(\cdot)\| \cdot \lambda$. Since $\omega \mapsto 1 /\|H(\omega)\|$ is continuous and strictly positive on $\Omega$, it follows that $G:=H(\cdot) /\|H(\cdot)\|$ is continuous on $\Omega$ and so has compact range in $S[X]$. In particular, $G=d m / d|m|$ is Bochner $|m|$-integrable. Theorem 1 ensures that $I_{m}$ is compact.

Proof of Theorem 3. Let $W:=\left\{y_{n}: n \geq 2\right\} \subseteq S[X]$ be a set which is not relatively compact but is w-seminorm dominated; see Lemma 2.5. Let $\Omega:=\mathbb{N}$ and $\Sigma:=2^{\mathbb{N}}$, and define a vector measure $m: \Sigma \rightarrow X$ by $m(E)=\sum_{n \in E} 2^{-n} y_{n+1}$ for $E \in \Sigma$. Then $m$ has finite variation given by $|m|(E)=\sum_{n \in E} 2^{-n}$ for $E \in \Sigma$. Define $G: \Omega \rightarrow X$ by $G(n):=y_{n+1}$ for $n \in \Omega$. Since $\left\langle G(\cdot), x^{\prime}\right\rangle$ is $\Sigma$-measurable for each $x^{\prime} \in X^{\prime}\left(\right.$ as $\left.\Sigma=2^{\mathbb{N}}\right)$ and $G$ takes its values in the separable subspace of $X$ generated by $W$, the Pettis measurability theorem ensures that $G$ is strongly measurable. Moreover, $\int_{\Omega}\|G(\cdot)\| d|m|=|m|(\Omega)<\infty$ and so $G \in \mathcal{B}(|m|, X)$. It is routine to verify that $m=G \cdot|m|$ and so $d m / d|m|=G \in \mathcal{B}(|m|, X)$. Since $G(\Omega)=W$ is wseminorm dominated, Lemma 2.6 implies that $L^{1}(m)=L^{1}(|m|)$. But $G$ does not have $|m|$-essentially relatively compact range in $X$ and so Theorem 1 shows that $I_{m}$ is not compact.

Proof of Theorem 4. Denote the domain of $m$ by $\Sigma$ and let $\lambda: \Sigma \rightarrow$ $[0, \infty)$ be a control measure for $m$. That is, $\lambda$ is a finite measure such that $\lambda(E) \rightarrow 0$ implies $m(E) \rightarrow 0$, or equivalently $\lambda(E)=0$ implies $m(E)=0$; 
see [6, p. 10 \& p. 14]. By Rybakov's theorem [6, Theorem IX.2.2], it is possible to choose (which we do) $\lambda=\left|\left\langle m, x_{0}^{\prime}\right\rangle\right|$ for a suitable $x_{0}^{\prime} \in X^{\prime}$. It is then clear that

$$
L^{\infty}(m)=L^{\infty}(\lambda) \subseteq L^{1}(m) \subseteq L^{1}(\lambda) .
$$

Moreover, if we define $\psi_{x^{\prime}}:=d\left\langle m, x^{\prime}\right\rangle / d \lambda$ for $x^{\prime} \in X^{\prime}$, then $\psi_{x^{\prime}} \in L^{1}(\lambda)$ and

$$
\|f\|_{m}=\sup \left\{\int_{\Omega}|f| \cdot\left|\psi_{x^{\prime}}\right| d \lambda: x^{\prime} \in B\left[X^{\prime}\right]\right\}, \quad f \in L^{1}(m) .
$$

Suppose that $m: \Sigma \rightarrow X$ does not have finite variation. Then for every $h \in L^{1}(\lambda)$ there exists $A \in \Sigma$ (depending on $h$ ) such that $\|m(A)\|>$ $\int_{A}|h| d \lambda$. It is to be proved that $I_{m}$ is not compact. To this end we construct, inductively, a sequence $\left\{g_{n}\right\}_{n=1}^{\infty} \subseteq B\left[L^{1}(m)\right]$ such that $\left\|I_{m}\left(g_{n}\right)-I_{m}\left(g_{k}\right)\right\|$ $\geq 1 / 4$ whenever $n \neq k$.

Choose $g_{1} \in B\left[L^{1}(m)\right]$ arbitrarily and suppose that functions $g_{1}, \ldots, g_{n}$ $\in B\left[L^{1}(m)\right]$ have been constructed with the stated property. Let $H \subseteq X$ be the finite-dimensional subspace spanned by $\left\{I_{m}\left(g_{1}\right), \ldots, I_{m}\left(g_{n}\right)\right\}$. By Lemma 2.8 with $\varepsilon:=1 / 4, Y:=X^{\prime}$ and $Z:=H^{\perp}=\left\{x^{\prime} \in X^{\prime}:\left\langle I_{m}\left(g_{j}\right), x^{\prime}\right\rangle=0\right.$ for $1 \leq j \leq n\}$, there is a finite set $F=\left\{x_{1}^{\prime}, \ldots, x_{N}^{\prime}\right\}$ in $B\left[X^{\prime}\right]$ with $N \in \mathbb{N}$ such that

$$
B\left[X^{\prime}\right] \subseteq \frac{5}{4}\left(\mathrm{bco}(F)+2 B\left[H^{\perp}\right]\right) .
$$

Define $\psi$ by $\omega \mapsto \psi(\omega):=\max \left\{\left|\psi_{x_{j}^{\prime}}(\omega)\right|: 1 \leq j \leq N\right\}$ for $\omega \in \Omega$. Since $5 \psi \in L^{1}(\lambda)$ and $|m|(\Omega)=\infty$, we noted above that there is a set $A \in \Sigma$ with $\|m(A)\|>5 \int_{A} \psi d \lambda$. Then $\alpha:=\left\|\chi_{A}\right\|_{m}$ satisfies

$$
\alpha \geq\|m(A)\|>5 \int_{A} \psi d \lambda \geq 0 .
$$

Moreover, $\alpha=\sup \left\{\int_{A}\left|\psi_{u^{\prime}}\right| d \lambda: u^{\prime} \in B\left[X^{\prime}\right]\right\}$ by (3.2), and so we can choose $x^{\prime} \in B\left[X^{\prime}\right]$ such that $\frac{7}{8} \alpha<\int_{A}\left|\psi_{x^{\prime}}\right| d \lambda$. By (3.3) there exist complex numbers $\alpha_{j}$, for $1 \leq j \leq N$, with $\sum_{j=1}^{N}\left|\alpha_{j}\right| \leq 1$ and $z^{\prime} \in B\left[H^{\perp}\right]$ such that $x^{\prime}=$ $\frac{5}{4}\left(\sum_{j=1}^{N} \alpha_{j} x_{j}^{\prime}+2 z^{\prime}\right)$. Then $\psi_{x^{\prime}}=\frac{5}{4}\left(\sum_{j=1}^{N} \alpha_{j} \psi_{x_{j}^{\prime}}+2 \psi_{z^{\prime}}\right)$ satisfies $\left|\psi_{x^{\prime}}\right| \leq$ $\frac{5}{4}\left(\psi+2\left|\psi_{z^{\prime}}\right|\right)$ and hence

$$
\frac{7}{8} \alpha<\frac{5}{4} \int_{A} \psi d \lambda+\frac{5}{2} \int_{A}\left|\psi_{z^{\prime}}\right| d \lambda<\frac{\alpha}{4}+\frac{5}{2} \int_{A}\left|\psi_{z^{\prime}}\right| d \lambda .
$$

It follows that $\alpha / 4<\int_{A}\left|\psi_{z^{\prime}}\right| d \lambda$. Now define

$$
g_{n+1}:=\alpha^{-1} \chi_{A} \cdot\left|\psi_{z^{\prime}}\right| / \psi_{z^{\prime}}
$$

with the understanding that $0 / 0=1$, and note that $g_{n+1} \in L^{\infty}(\lambda)=$ $L^{\infty}(m) \subseteq L^{1}(m)$. Moreover, $\left|g_{n+1}\right|=\alpha^{-1} \chi_{A}$, from which it follows that $\left\|g_{n+1}\right\|_{m}=1$, i.e., $g_{n+1} \in B\left[L^{1}(m)\right]$. Now fix $k \in\{1, \ldots, n\}$. Then we have 
$\left\langle I_{m}\left(g_{k}\right), z^{\prime}\right\rangle=0$, since $z^{\prime} \in H^{\perp}$. Accordingly, since also $\left\|z^{\prime}\right\| \leq 1$, we see that

$$
\begin{aligned}
\left\|I_{m}\left(g_{n+1}\right)-I_{m}\left(g_{k}\right)\right\| & \geq\left|\left\langle I_{m}\left(g_{n+1}\right)-I_{m}\left(g_{k}\right), z^{\prime}\right\rangle\right|=\left|\left\langle I_{m}\left(g_{n+1}\right), z^{\prime}\right\rangle\right| \\
& =\left|\int_{\Omega} \psi_{z^{\prime}} g_{n+1} d \lambda\right|=\alpha^{-1} \int_{A}\left|\psi_{z^{\prime}}\right| d \lambda>\frac{1}{4} .
\end{aligned}
$$

This completes the construction of $g_{n+1}$, and hence also the proof.

Acknowledgments. The authors wish to thank Professors J. Forbes and J. Giles for useful discussions and suggestions.

\section{References}

[1] G. P. Curbera, Operators into $L^{1}$ of a vector measure and applications to Banach lattices, Math. Ann. 293 (1992), 317-330.

[2] - When is $L^{1}$ of a vector measure an AL-space?, Pacific J. Math. 162 (1994), 287-303.

[3] -, Banach space properties of $L^{1}$ of a vector measure, Proc. Amer. Math. Soc. 123 (1995), 3797-3806.

[4] - , Volterra convolution operators with values in rearrangement invariant spaces, J. London Math. Soc. (2) 60 (1999), 258-268.

[5] J. Diestel, Sequences and Series in Banach Spaces, Springer, New York, 1984.

[6] J. Diestel and J. J. Uhl Jr., Vector Measures, Math. Surveys 15, Amer. Math. Soc., Providence, 1977.

[7] N. Dinculeanu, Vector Measures, Pergamon Press, London, 1967.

[8] P. G. Dodds and W. J. Ricker, Spectral measures and the Bade reflexivity theorem, J. Funct. Anal. 61 (1985), 136-163.

[9] L. Drewnowski and Z. Lipecki, On vector measures which have everywhere infinite variation or noncompact range, Dissertationes Math. 334 (1995).

[10] D. van Dulst, Characterizations of Banach Spaces not Containing $\ell^{1}$, CWI Tract 59, Centrum voor Wiskunde en Informatica, Amsterdam, 1989.

[11] N. Dunford and J. T. Schwartz, Linear Operators I: General Theory, Wiley-Interscience, New York, 1966.

[12] L. Janicka and N. J. Kalton, Vector measures of infinite variation, Bull. Acad. Polon. Sci. Sér. Sci. Math. Astronom. Phys. 25 (1977), 239-241.

[13] H. Jarchow, Locally Convex Spaces, Teubner, Stuttgart, 1981.

[14] I. Kluvánek and G. Knowles, Vector Measures and Control Systems, North-Holland, Amsterdam, 1976.

[15] D. R. Lewis, Integration with respect to a vector measure, Pacific J. Math. 33 (1970), 157-165.

[16] —, On integrability and summability in vector spaces, Illinois J. Math. 16 (1972), 294-307.

[17] S. Okada, The dual space of $L^{1}(\mu)$ for a vector measure $\mu$, J. Math. Anal. Appl. 177 (1993), 583-599.

[18] S. Okada and W. J. Ricker, Non-weak compactness of the integration map associated with a vector measure, J. Austral. Math. Soc. Ser. A 54 (1993), 287-303.

[19] - - - Compactness properties of the integration map associated with a vector measure, Colloq. Math. 66 (1994), 175-185. 
[20] W. J. Ricker, Compactness properties of extended Volterra operators in $L^{p}([0,1])$ for $1 \leq p \leq \infty$, Arch. Math. (Basel) 66 (1996), 132-140.

[21] L. Rodríguez-Piazza, Derivability, variation and range of a vector measure, Studia Math. 112 (1995), 165-187.

[22] L. Rodríguez-Piazza and M. C. Romero-Moreno, Conical measures and properties of a vector measure determined by its range, ibid. 125 (1997), 255-270.

[23] E. Thomas, The Lebesgue-Nikodým theorem for vector valued Radon measures, Mem. Amer. Math. Soc. 139 (1974).

S. Okada

School of Mathematics

The University of New South Wales

Sydney, NSW 2052, Australia

Current address:

Department of Mathematics

Macquarie University

Sydney, NSW 2109, Australia

E-mail: okada@maths.mq.edu.au

L. Rodríguez-Piazza

Departamento de Análisis Matemático

Facultad de Matemáticas

Universidad de Sevilla

Aptdo. 1160

41080 Sevilla, Spain

E-mail: piazza@cica.es
W. J. Ricker

School of Mathematics The University of New South Wales Sydney, NSW 2052, Australia

Current address:

Math.-Geogr. Fakultät

Katholische Universität Eichstätt

D-85071 Eichstätt, Germany

E-mail: werner.ricker@ku-eichstaett.de

Received December 15, 2000

Revised version December 27, 2001 